\title{
ON A CONSTANT ARISING IN MANIN'S CONJECTURE FOR DEL PEZZO SURFACES
}

\author{
Ulrich Derenthal
}

\begin{abstract}
For split smooth Del Pezzo surfaces, we analyse the structure of the effective cone and prove a recursive formula for the value of $\alpha$, appearing in the leading constant as predicted by Peyre of Manin's conjecture on the number of rational points of bounded height on the surface. Furthermore, we calculate $\alpha$ for all singular Del Pezzo surfaces of degree $\geq 3$.
\end{abstract}

\section{Introduction}

Over the field $\mathbb{Q}$ of rational numbers, a split smooth Del Pezzo surface $S$ is $\mathbb{P}^{2}$, $\mathbb{P}^{1} \times \mathbb{P}^{1}$, or, for $1 \leq r \leq 8$, the blow-up $S_{r}$ of $\mathbb{P}^{2}$ in $r$ points which are defined over $\mathbb{Q}$ and are in general ${ }^{1}$ position. For the degree of $S$, we have $\operatorname{deg}\left(\mathbb{P}^{2}\right)=9, \operatorname{deg}\left(\mathbb{P}^{1} \times \mathbb{P}^{1}\right)=8$, and $\operatorname{deg}\left(S_{r}\right)=9-r$.

An important object associated to $S$ is the effective cone $\Lambda_{\text {eff }}(S)$, i.e., the convex cone in

$$
\operatorname{Pic}(S)_{\mathbb{R}}:=\operatorname{Pic}(S) \otimes_{\mathbb{Z}} \mathbb{R}
$$

which is generated by the classes of effective divisors. Note that we identify divisors and their classes in $\operatorname{Pic}(S)$ when this cannot lead to confusion.

On $\operatorname{Pic}(S) \cong \mathbb{Z}^{10-\operatorname{deg}(S)}$, we have the intersection form $(\cdot, \cdot)$, which is a nondegenerate symmetric bilinear form. The anticanonical map $\phi: S \rightarrow \mathbb{P}^{\operatorname{deg}(S)}$ is given by the anticanonical class $-K_{S}=\phi^{*}\left(\mathcal{O}_{\mathbb{P d e g}(S)}(1)\right)$. It is an embedding for $\operatorname{deg}(S) \geq 3$.

For $S=S_{r}$, the anticanonical class is

$$
-K_{r}:=-K_{S_{r}}=3 H-\left(E_{1}+\cdots+E_{r}\right),
$$

where $H$ is the transform of a general line in $\mathbb{P}^{2}$, and $E_{1}, \ldots, E_{r}$ are the exceptional divisors obtained by blowing up the $r$ points in $\mathbb{P}^{2}$. By [2, Corollary 3.3], for $r \geq 2$, $\Lambda_{\text {eff }}\left(S_{r}\right)$ is generated by the (-1)-curves, i.e., prime divisors $D$ whose self-intersection number $(D, D)$ is -1 . For $r \leq 6$, the anticanonical embedding $\phi_{r}=\phi$ maps the $(-1)$-curves on $S_{r}$ exactly to the lines on $\phi_{r}\left(S_{r}\right) \subset \mathbb{P}^{9-r}$. Note that $H, E_{1}, \ldots, E_{r}$ give a basis of $\operatorname{Pic}\left(S_{r}\right) \cong \mathbb{Z}^{r+1}$.

Starting in 1989, Manin initiated a program to study the number of rational points on certain varieties which can be stated in case of a split smooth Del Pezzo surface $S$ of degree $d$ as follows:

Received by the editors March 24, 2006.

2000 Mathematics Subject Classification. Primary 14J26; Secondary 52B05, 14 G05.

Key words and phrases. Del Pezzo surface, effective cone, Manin's conjecture.

${ }^{1}$ I.e., no three points on a line, no six on a conic, no eight on a cubic curve with one of them a singularity of that curve. 
Question 1. Let $U$ be the complement of the (-1)-curves in $S$, and let

$$
H: S(\mathbb{Q}) \rightarrow \mathbb{Z}_{>0}
$$

be the anticanonical height, i.e., $H(\mathbf{x})=\max \left\{\left|x_{0}\right|, \ldots,\left|x_{d}\right|\right\}$ where the image in $\mathbb{P}^{d}(\mathbb{Q})$ of $\mathbf{x} \in S(\mathbb{Q})$ under the anticanonical map $\phi: S \rightarrow \mathbb{P}^{d}$ is represented by integral and coprime coordinates $\left(x_{0}, \ldots, x_{d}\right)$.

What is the asymptotic behavior of the number of rational points of bounded height

$$
N_{U, H}(B):=\#\{\mathbf{x} \in U(\mathbb{Q}) \mid H(\mathbf{x}) \leq B\}
$$

as $B \rightarrow \infty$ ?

By Manin's conjecture [15], which was formulated for the much larger class of Fano varieties, the following answer is expected:

$$
N_{U, H}(B) \sim c_{S, H} \cdot B \cdot(\log B)^{9-d} .
$$

The leading constant $c_{S, H}$ has received a conjectural interpretation by Peyre [18]: it is expected to be the product

$$
c_{S, H}=\alpha(S) \cdot \beta(S) \cdot \omega_{H}(S),
$$

where $\alpha(S)$ is a constant related to the geometry of $S$, while $\beta(S)$ is a cohomological constant which is always 1 for split Del Pezzo surfaces, and $\omega_{H}(S)$ is related to the densities of rational points on $S$ over $\mathbb{R}$ and modulo $p$ for all primes $p$.

So far, Manin's conjecture has been proved for split smooth Del Pezzo surfaces in the cases $d \geq 6$ in the context of the more general proof for toric varieties [3], and for a specific surface in the case $d=5[4]$.

The purpose of this note is to look more closely at the constant $\alpha(S)$. Its definition is due to Peyre ([18, Definition 2.4]; see [19, Section 6] for more details):

Definition 2. Let $\Lambda_{\text {eff }}(S)$ be the effective cone, $\Lambda_{\text {eff }}^{\vee}(S)$ its dual cone (with respect to the intersection form) of nef divisor classes, and $-K_{S}$ the anticanonical class on $S$. Then we define

$$
\alpha(S):=\operatorname{Vol}(P(S)),
$$

where

$$
P(S):=\left\{x \in \Lambda_{\text {eff }}^{\vee}(S) \mid\left(-K_{S}, x\right)=1\right\}
$$

is a polytope whose volume is calculated using the Lebesgue measure on the hyperplane $\left\{x \in \operatorname{Pic}(S)_{\mathbb{R}}^{\vee} \mid\left(-K_{S}, x\right)=1\right\}$ which is defined by the $(9-d)$-form $\mathrm{d} \mathbf{x}$ such that $\mathrm{d} \mathbf{x} \wedge \mathrm{d} \omega=\mathrm{d} \mathbf{y}$, where $\mathrm{d} \mathbf{y}$ is the form corresponding to the natural Lebesgue measure on $\operatorname{Pic}(S)_{\mathbb{R}}^{\vee}$ and $\mathrm{d} \omega$ is the linear form defined by $-K_{S}$ on $\operatorname{Pic}(S)_{\mathbb{R}}^{\vee}$.

For large $d$, the calculation of $\alpha(S)$ can be carried out directly by hand (see [4, Section 1.3] for the case $d=5$ ). For small $d$, especially for $S_{8}$ of degree $d=1$, a direct calculation seems to be currently impossible even with the help of software like Polymake [17]. In this case, the cone $\Lambda_{\text {eff }}\left(S_{8}\right)$ has 240 generators, while $\Lambda_{\text {eff }}^{\vee}\left(S_{8}\right)$ has 19440 generators. A direct calculation of $\alpha\left(S_{8}\right)$ would require a triangulation of $\Lambda_{\text {eff }}^{\vee}\left(S_{8}\right)$, which seems to be out of reach for today's software and hardware.

Therefore, we need a more detailed knowledge of $\Lambda_{\text {eff }}^{\vee}(S)$. For $S=S_{r}$ and $r \geq 3$, we have an action of a Weyl group $W_{r}$ on $\operatorname{Pic}\left(S_{r}\right)$; see Table 1 for the type of $W_{r}$ and [2, Section 2] for details. Our main result which will allow us to compute $\alpha\left(S_{r}\right)$ recursively is: 
Theorem 3. Let $r \geq 3$. The nef cone $\Lambda_{\mathrm{eff}}^{\vee}\left(S_{r}\right)$ has $N_{r}$ faces, where $N_{r}$ is the number of $(-1)$-curves on $S_{r}$. Each face is isomorphic to $\Lambda_{\text {eff }}^{\vee}\left(S_{r-1}\right)$. The Weyl group $W_{r}$ acts transitively on the faces and leaves $-K_{r}$ in the interior of $\Lambda_{\mathrm{eff}}^{\vee}\left(S_{r}\right)$ fixed.

This observation is a crucial step in the proof of the following recursive formula for $\alpha\left(S_{r}\right)$; see Table 1 for the values of $\alpha\left(S_{r}\right)$ and $N_{r}$ :

Theorem 4. Let $S_{r}$ be a split smooth Del Pezzo surface of degree $9-r$ which is the blow-up of $\mathbb{P}^{2}$ in $r$ points in general position. Let $N_{r}$ be the number of $(-1)$-curves on $S_{r}$. We have $\alpha\left(S_{2}\right)=1 / 24$ and

$$
\alpha\left(S_{r}\right)=\frac{N_{r} \cdot \alpha\left(S_{r-1}\right)}{r \cdot(9-r)}
$$

for $3 \leq r \leq 8$. Furthermore, $\alpha\left(S_{1}\right)=1 / 6, \alpha\left(\mathbb{P}^{1} \times \mathbb{P}^{1}\right)=1 / 4$, and $\alpha\left(\mathbb{P}^{2}\right)=1 / 3$.

\begin{tabular}{|c||c|c|c|c|c|c|c|}
\hline$r$ & 2 & 3 & 4 & 5 & 6 & 7 & 8 \\
\hline \hline type of $W_{r}$ & & $\mathbf{A}_{2} \times \mathbf{A}_{1}$ & $\mathbf{A}_{4}$ & $\mathbf{D}_{5}$ & $\mathbf{E}_{6}$ & $\mathbf{E}_{7}$ & $\mathbf{E}_{8}$ \\
\hline$N_{r}$ & 3 & 6 & 10 & 16 & 27 & 56 & 240 \\
\hline$\alpha\left(S_{r}\right)$ & $1 / 24$ & $1 / 72$ & $1 / 144$ & $1 / 180$ & $1 / 120$ & $1 / 30$ & 1 \\
\hline
\end{tabular}

TABle 1. Smooth Del Pezzo surfaces

Next, we consider split singular Del Pezzo surfaces $S$ whose singularities are rational double points. Besides the case where $S$ is the Hirzebruch surface $F_{2}$ of degree 8 , their minimal desingularizations $\widetilde{S}$ are obtained as follows: we perform a series of $r \leq 8$ blow-ups of $\mathbb{P}^{2}$ resulting in $\widetilde{S}=\widetilde{S}_{r}$, where in at least one step, we blow up a point on a $(-1)$-curve (resulting in $(-2)$-curves, i.e., prime divisors with self intersection number -2 ), where the only restriction for the choice of the blown-up point is that we never blow up a point on a $(-2)$-curve (therefore, no prime divisors with self intersection number smaller than -2 can occur). Contracting the $(-2)$-curves on $\widetilde{S}_{r}$ results in $S=S_{r}$ of degree $9-r$.

With some minor modifications in its formulation (mostly replacing $S$ by $\widetilde{S}$ where appropriate), Manin's conjecture is expected to hold for singular Del Pezzo surfaces as well; see [9] and the introduction of [14] for details and an overview of the current progress. The definition of $\alpha(S)$ is also almost the same: we must consider $-K_{\widetilde{S}}$, $\Lambda_{\text {eff }}(\widetilde{S})$, and $\Lambda_{\text {eff }}^{\vee}(\widetilde{S})$ in $\operatorname{Pic}(\widetilde{S})$ of rank $10-\operatorname{deg}(S)$. Note that $\Lambda_{\text {eff }}(\widetilde{S})$ is generated by the negative curves (i.e., the $(-1)$ - and $(-2)$-curves) in the singular case if $\operatorname{deg}(S) \leq 7$. The value of $\alpha(S)$ depends not only on the degree of $S$, but also on the type of singularities on $S$.

In Section 3, we list $\alpha(S)$ for each type of singular Del Pezzo surface of degree at least 3; see Tables 2, 3, 4, 5, and 6. For most types, the calculation was performed with the help of the data given in [12] and [13, Chapter 6].

For some examples of the calculation of $\alpha\left(S_{r}\right)$ for non-split Del Pezzo surfaces, see $[18],[19$, Section 6], [8], and [6]. 


\section{Smooth Del Pezzo surfaces}

Let $S_{r}$ be the blow-up of $\mathbb{P}^{2}$ in $r$ points in general position.

Lemma 5. Let $2 \leq r \leq 8$. The effective cone $\Lambda_{\text {eff }}\left(S_{r}\right)$ is generated over $\mathbb{R}$ by the (-1)-curves, whose number is $N_{r}$ as listed in Table 1.

Proof. See [2, Corollary 3.3]. Their number $N_{r}$ can be found in [2, Theorem 2.1]. (Note that, for $r=8$, the semigroup of classes of effective divisors is generated by the $(-1)$-curves together with $-K_{8}$.)

Lemma 6. Let $E$ be a (-1)-curve on $S_{r}$ for $r \geq 3$. If $D \in \operatorname{Pic}\left(S_{r}\right)$ fulfills $(D, E)=0$ and $\left(D, E^{\prime}\right) \geq 0$ for all $(-1)$-curves $E^{\prime}$ such that $\left(E, E^{\prime}\right)=0$, then $D$ is nef.

Proof. As the (-1)-curves generate the effective cone, we must show that $\left(D, E^{\prime}\right) \geq 0$ also holds for all $(-1)$-curves $E^{\prime}$, regardless of the value of $\left(E, E^{\prime}\right)$.

If $\left(E, E^{\prime}\right)<0$, then $E^{\prime}=E$, and $(D, E)=0$. If $\left(E, E^{\prime}\right)=0$, then $\left(D, E^{\prime}\right) \geq 0$ by assumption.

We proceed by induction on $n=\left(E, E^{\prime}\right)$. If $n=1$, then $E+E^{\prime}$ is a ruling as in [2, Definition 4.6]. The case $n=2$ occurs for $r \in\{7,8\}$, and $n=3$ is possible only for $r=8$; furthermore, $n \geq 4$ is impossible. The divisor $E+E^{\prime}$ can be written in at least two ways as the sum of two negative curves (see [2, Section 4] for rulings, and [13, Sections 3.4, 3.5] for $n \in\{2,3\})$, say $E+E^{\prime}=E_{1}+E_{2}$, where $E \notin\left\{E_{1}, E_{2}\right\}$. Then

$$
\left(E, E_{1}\right)+\left(E, E_{2}\right)=\left(E, E^{\prime}\right)+(E, E)=n-1,
$$

where $\left(E, E_{1}\right)$ and $\left(E, E_{2}\right)$ are both non-negative. Therefore, the induction hypothesis holds for $E_{1}, E_{2}$, and

$$
\left(D, E^{\prime}\right)=\left(D, E+E^{\prime}\right)=\left(D, E_{1}+E_{2}\right)=\left(D, E_{1}\right)+\left(D, E_{2}\right) \geq 0
$$

completes the induction.

For any $D \in \operatorname{Pic}\left(S_{r}\right)$ and $c \in \mathbb{R}$, we define

$$
D^{=c}:=\left\{D^{\prime} \in \operatorname{Pic}\left(S_{r}\right)_{\mathbb{R}} \mid\left(D, D^{\prime}\right)=c\right\},
$$

and similarly,

$$
D^{\geq c}:=\left\{D^{\prime} \in \operatorname{Pic}\left(S_{r}\right)_{\mathbb{R}} \mid\left(D, D^{\prime}\right) \geq c\right\} .
$$

Proof of Theorem 3. By definition, $\Lambda_{\text {eff }}^{\vee}\left(S_{r}\right)$ is the intersection of the half spaces $E^{\geq 0}$ for all generators $E$ of $\Lambda_{\mathrm{eff}}\left(S_{r}\right)$, which are exactly the (-1)-curves by Lemma 5 . By [16, Lemma 5.3], $W_{r}$ acts transitively on the $(-1)$-curves. This symmetry implies that each (-1)-curve $E$ defines a proper face $F_{E}:=\Lambda_{\text {eff }}\left(S_{r}\right) \cap E^{=0}$, and that $W_{r}$ acts transitively on the set of faces $\left\{F_{E} \mid E\right.$ is a $(-1)$-curve $\}$.

Consider $S_{r}$ as the blow-up of $S_{r-1}$ in one point, resulting in the exceptional divisor $E_{r}$. Then

$$
\operatorname{Pic}\left(S_{r}\right)=\operatorname{Pic}\left(S_{r-1}\right) \oplus \mathbb{Z} \cdot E_{r}
$$

is an orthogonal sum.

We claim that $F_{E_{r}}=\Lambda_{\text {eff }}\left(S_{r-1}\right)$, where we regard $\Lambda_{\text {eff }}\left(S_{r-1}\right) \subset \operatorname{Pic}\left(S_{r-1}\right)$ as embedded into $\operatorname{Pic}\left(S_{r}\right)$. 
Indeed, if $D \in \Lambda_{\text {eff }}\left(S_{r-1}\right)$, then $\left(D, E_{r}\right)=0$, and $(D, E) \geq 0$ for all $(-1)$-curves $E$ of $S_{r-1}$, which are exactly the $(-1)$-curves of $S_{r}$ with $\left(E, E_{r}\right)=0$. By Lemma 6 , we have $(D, E) \geq 0$ for all $(-1)$-curves of $S_{r}$.

On the other hand, if $D \in E_{r}^{=0}$, then $D \in \operatorname{Pic}\left(S_{r-1}\right)$. If $D \in \Lambda_{\text {eff }}\left(S_{r}\right)$, then $(D, E) \geq 0$ for all $(-1)$-curves of $S_{r}$, which includes the (-1)-curves of $S_{r-1}$, proving the other direction.

The root system corresponding to $W_{r}$ is

$$
R_{r}=\left\{D \in \operatorname{Pic}\left(S_{r}\right) \mid(D, D)=-2,\left(D,-K_{r}\right)=0\right\} .
$$

Since $W_{r}$ is generated by the reflections $E \mapsto E+(D, E) \cdot D$ corresponding to the roots $D \in R_{r}$, the anticanonical class $-K_{r}$ is fixed under $W_{r}$. This completes the proof of Theorem 3 .

Proof of Theorem 4. The polytope $P_{r}:=P\left(S_{r}\right)$ whose volume is $\alpha\left(S_{r}\right)$ (see Definition 2) is the intersection of the $N_{r}$ half-spaces $E^{\geq 0}$ (where $E$ runs through the $(-1)$-curves of $S_{r}$ ) in the $r$-dimensional space $-K_{r}^{=1}$.

Note that $\left(-K_{r},-K_{r}\right)=9-r$. Therefore, $Q:=\frac{1}{9-r} \cdot\left(-K_{r}\right) \in-K_{r}^{=1}$, and since $\left(-K_{r}, E\right)=1$ for any $(-1)$-curve $E$, the point $Q$ is in the interior of $P_{r}$.

Consider the convex hull $P_{E}$ of $Q$ and the face $P_{r} \cap E^{=0}$ of $P_{r}$ corresponding to $E$. Then $P_{r}$ is the union of the $P_{E}$ for all $(-1)$-curves $E$, and since their intersections are lower-dimensional,

$$
\operatorname{Vol}\left(P_{r}\right)=\sum_{E} \operatorname{Vol}\left(P_{E}\right)
$$

As the intersection form and $-K_{r}$ are invariant under the Weyl group $W_{r}$, it acts on $-K_{r}^{=1}$ and therefore on $P_{r}$. As in Theorem 3, it permutes the faces of $P_{r}$ transitively. As $Q$ is fixed under $W_{r}$ and the volume is invariant under $W_{r}$, we have $\operatorname{Vol}\left(P_{r}\right)=N_{r} \cdot \operatorname{Vol}\left(P_{E}\right)$ for any $(-1)$-curve $E$.

As in the proof of Theorem 3, we consider $S_{r}$ as the blow-up of $S_{r-1}$ in one point, resulting in the exceptional divisor $E_{r}$, with the orthogonal sum

$$
\operatorname{Pic}\left(S_{r}\right)=\operatorname{Pic}\left(S_{r-1}\right) \oplus \mathbb{Z} \cdot E_{r} .
$$

We claim that $P_{r} \cap E_{r}^{=0}=P_{r-1}$. In view of Theorem 3, it remains to prove that $\left(D,-K_{r-1}\right)=1$ is equivalent to $\left(D,-K_{r}\right)=1$ on $E_{r}^{=0}$. This follows directly from $-K_{r}=-K_{r-1}-E_{r}$.

Therefore, $P_{E_{r}}$ is a cone over the $(r-1)$-dimensional polytope $P_{r-1}$ in the $r$ dimensional space $-K_{r}^{=1}$. A cone of height 1 over $P_{r-1}$ has volume $\operatorname{Vol}\left(P_{r-1}\right) / r$. As $E_{r}$ is orthonormal to $\operatorname{Pic}\left(S_{r-1}\right)$, and $\left(-K_{r}, E_{r}\right)=1$, the distance of $Q$ to $P_{r-1}$ is $1 /(9-r)$. Therefore,

$$
\operatorname{Vol}\left(P_{E_{r}}\right)=\frac{\operatorname{Vol}\left(P_{r-1}\right)}{r \cdot(9-r)} .
$$

Together with $\alpha\left(S_{r-1}\right)=\operatorname{Vol}\left(P_{r-1}\right)$ and $\operatorname{Vol}\left(P_{r}\right)=N_{r} \cdot \operatorname{Vol}\left(P_{E_{r}}\right)$, this completes the proof of the recursive formula. 
For $r=2$, we have $\Lambda_{\text {eff }}=\left\langle E_{1}, E_{2}, H-E_{1}-E_{2}\right\rangle$ and $-K_{2}=3 H-E_{1}-E_{2}$. Therefore, $\alpha\left(S_{2}\right)$ is the volume of

$$
\begin{aligned}
& \left\{\left(a_{0}, a_{1}, a_{2}\right) \in \mathbb{R}^{3} \mid 3 a_{0}-a_{1}-a_{2}=1, a_{1} \geq 0, a_{2} \geq 0, a_{0}-a_{1}-a_{2} \geq 0\right\} \\
= & \left\{\left(a_{0}, a_{1}\right) \in \mathbb{R}^{2} \mid a_{1} \geq 0,3 a_{0}-a_{1}-1 \geq 0,-2 a_{0}+1 \geq 0\right\} \\
= & \text { convex hull of }(1 / 3,0),(1 / 2,0),(1 / 2,1 / 2),
\end{aligned}
$$

which is a rectangular triangle whose legs have length $1 / 6$ and $1 / 2$. Hence, $\alpha\left(S_{2}\right)=$ $1 / 24$, while $\alpha\left(S_{1}\right)=1 / 6, \alpha\left(\mathbb{P}^{1} \times \mathbb{P}^{1}\right)=1 / 4$, and $\alpha\left(\mathbb{P}^{2}\right)=1 / 3$ can also be calculated directly, which completes the proof of Theorem 4 .

Remark 7. By the proof of $\left[18\right.$, Lemme 9.4.2], $\alpha\left(S_{1}\right)=1 / 6$, and by the proof of $[18$, Lemme 10.4.2],

$$
\alpha\left(S_{2}\right)=1 / 3 \cdot \operatorname{Vol}\left\{\left(x_{1}, x_{2}\right) \in \mathbb{R}_{>0}^{2} \mid x_{1}+x_{2} \leq 1 / 2\right\},
$$

which is clearly $\alpha_{2}=1 / 24$ and therefore agrees with our result. Note that the recursion formula does not hold for $r=2$ :

$$
\alpha\left(S_{2}\right)=\frac{1}{24} \neq \frac{N_{2} \cdot \alpha\left(S_{1}\right)}{2 \cdot(9-2)}=\frac{1}{28} .
$$

The value $\alpha\left(S_{4}\right)=1 /(6 \cdot 4 !)$ was previously calculated in [4, Section 1.3].

\section{Singular Del Pezzo surfaces}

For the classification of singular Del Pezzo surfaces, see [10], [11], [1]. It turns out that in each degree, the surfaces can be divided into different types according to the number and types of their singularities and the number of lines.

For each type, we might have more than one isomorphism class (e.g., two for type $\mathbf{D}_{4}$ in degree 3, and an infinite family for type $\mathbf{A}_{1}$ in degree 3 ). However, the degrees in $\operatorname{Pic}\left(\widetilde{S}_{r}\right)$ of the negative curves, which generate the effective cone, and of $-K_{r}$ only depend on the type. Therefore, $\alpha\left(S_{r}\right)$ depends only on the type.

More precisely, all this information is encoded for each type in its extended Dynkin diagram of negative curves. For degree $\geq 4$, these diagrams can be found in [11, Propositions $6.1,8.1,8.3,8.4]$. In [1], the information for each degree is encoded in a smaller number of diagrams. Also see [12] and [13, Chapter 6] for detailed information on several singular types of various degrees.

From the extended Dynkin diagram, we can derive a basis of $\operatorname{Pic}\left(\widetilde{S}_{r}\right)$, and $-K_{r}$ and all effective divisors in terms of this basis as explained in [12, Section 3].

With this information, it is straightforward to calculate $\alpha\left(S_{r}\right)$ for minimal desingularizations of all split types of degree $\geq 3$. In practice, this task is significantly simplified by the use of software such as Polymake.

For the Hirzebruch surface $F_{2}$, which is the unique singular Del Pezzo surface of degree 8 , we have $\alpha\left(F_{2}\right)=1 / 8$.

For $S_{r}$ of types $\mathbf{D}_{4}$ and $\mathbf{D}_{5}$ of degree 4 and $\mathbf{E}_{6}$ of degree 3 , the constant $\alpha\left(S_{r}\right)$ has been calculated while proving Manin's conjecture for these surfaces; see [14], [5], [7], respectively. Note that in these three cases, the calculation of $\alpha\left(S_{r}\right)$ is particularly simple as the effective cone is simplicial, i.e., the number of generators of $\Lambda_{\text {eff }}$ equals the rank of $\operatorname{Pic}\left(\widetilde{S}_{r}\right)$. 


\begin{tabular}{|c||c|c|c|c|}
\hline type & singularities & lines & generators of $\Lambda_{\text {eff }}^{\vee}$ & $\alpha$ \\
\hline \hline 0 & - & 3 & 3 & $1 / 24$ \\
$i$ & $\mathbf{A}_{1}$ & 2 & 3 & $1 / 48$ \\
\hline
\end{tabular}

TABle 2. Del Pezzo surfaces of degree 7

\begin{tabular}{|c||c|c|c|c|}
\hline type & singularities & lines & generators of $\Lambda_{\text {eff }}^{\vee}$ & $\alpha$ \\
\hline \hline 0 & - & 6 & 5 & $1 / 72$ \\
$i$ & $\mathbf{A}_{1}$ & 4 & 5 & $1 / 144$ \\
$i i$ & $\mathbf{A}_{1}$ & 3 & 4 & $1 / 144$ \\
$i i i$ & $2 \mathbf{A}_{1}$ & 2 & 4 & $1 / 288$ \\
$i v$ & $\mathbf{A}_{2}$ & 2 & 4 & $1 / 432$ \\
$v$ & $\mathbf{A}_{2}+\mathbf{A}_{1}$ & 1 & 4 & $1 / 864$ \\
\hline
\end{tabular}

Table 3. Del Pezzo surfaces of degree 6

\begin{tabular}{|c||c|c|c|c|}
\hline type & singularities & lines & generators of $\Lambda_{\text {eff }}^{\vee}$ & $\alpha$ \\
\hline \hline 0 & - & 10 & 10 & $1 / 144$ \\
$i$ & $\mathbf{A}_{1}$ & 7 & 9 & $1 / 288$ \\
$i i$ & $2 \mathbf{A}_{1}$ & 5 & 8 & $1 / 576$ \\
$i i i$ & $\mathbf{A}_{2}$ & 4 & 7 & $1 / 864$ \\
$i v$ & $\mathbf{A}_{2}+\mathbf{A}_{1}$ & 3 & 7 & $1 / 1728$ \\
$v$ & $\mathbf{A}_{3}$ & 2 & 5 & $1 / 3456$ \\
$v i$ & $\mathbf{A}_{4}$ & 1 & 5 & $1 / 17280$ \\
\hline
\end{tabular}

TABle 4. Del Pezzo surfaces of degree 5

Remark 8. We have two different types with the same singularities in three cases $\left(\mathbf{A}_{1}\right.$ in degree 6, 2 $\mathbf{A}_{1}$ and $\mathbf{A}_{3}$ in degree 4). The two types in each pair can be distinguished by their number of lines. Therefore, the two different types have different effective cones. However, both types in each pair have the same constant $\alpha$. It is unclear whether this is more than a coincidence.

Remark 9. For singular Del Pezzo surfaces $S_{r}$ of degree 2 and 1, we do not calculate $\alpha\left(S_{r}\right)$ since the number of different types is much larger than in degree $\geq 3$.

For surfaces of degree 1 whose effective cone has many generators, the triangulation of the nef cone might be too complicated for a computation of $\alpha$ using Polymake. We expect this to happen in case of "mild" singularities (e.g., of type $\mathbf{A}_{1}, \mathbf{A}_{2}$ or $2 \mathbf{A}_{1}$ ). Here, the nef cone should be almost as complicated as in the smooth case, and some help "by hand" might be necessary.

\section{Acknowledgements}

I am grateful to T. D. Browning for several useful comments. 


\begin{tabular}{|c||c|c|c|c|}
\hline type & singularities & lines & generators of $\Lambda_{\text {eff }}^{\vee}$ & $\alpha$ \\
\hline \hline 0 & - & 16 & 26 & $1 / 180$ \\
$i$ & $\mathbf{A}_{1}$ & 12 & 22 & $1 / 360$ \\
$i i$ & $2 \mathbf{A}_{1}$ & 9 & 19 & $1 / 720$ \\
$i i i$ & $2 \mathbf{A}_{1}$ & 8 & 17 & $1 / 720$ \\
$i v$ & $\mathbf{A}_{2}$ & 8 & 16 & $1 / 1080$ \\
$v$ & $3 \mathbf{A}_{1}$ & 6 & 15 & $1 / 1440$ \\
vi & $\mathbf{A}_{2}+\mathbf{A}_{1}$ & 6 & 15 & $1 / 2160$ \\
vii & $\mathbf{A}_{3}$ & 5 & 11 & $1 / 4320$ \\
viii & $\mathbf{A}_{3}$ & 4 & 10 & $1 / 4320$ \\
ix & $4 \mathbf{A}_{1}$ & 4 & 4 & $1 / 2880$ \\
$x$ & $\mathbf{A}_{2}+2 \mathbf{A}_{1}$ & 4 & 13 & $1 / 4320$ \\
xi & $\mathbf{A}_{3}+\mathbf{A}_{1}$ & 3 & 10 & $1 / 8640$ \\
xii & $\mathbf{A}_{4}$ & 3 & 9 & $1 / 21600$ \\
xiii & $\mathbf{D}_{4}$ & 2 & 6 & $1 / 34560$ \\
xiv & $\mathbf{A}_{3}+2 \mathbf{A}_{1}$ & 2 & 10 & $1 / 17280$ \\
xv & $\mathbf{D}_{5}$ & 1 & 6 & $1 / 345600$ \\
\hline
\end{tabular}

TABle 5. Del Pezzo surfaces of degree 4

\section{References}

[1] V. Alexeev and V. V. Nikulin, Del Pezzo and K3 surfaces, Vol. 15 of MSJ Memoirs, Mathematical Society of Japan, Tokyo (2006), ISBN 4-931469-34-5.

[2] V. V. Batyrev and O. N. Popov, The Cox ring of a del Pezzo surface, in Arithmetic of higher-dimensional algebraic varieties (Palo Alto, CA, 2002), Vol. 226 of Progr. Math., 85-103, Birkhäuser Boston, Boston, MA (2004).

[3] V. V. Batyrev and Y. Tschinkel, Manin's conjecture for toric varieties, J. Algebraic Geom. 7 (1998), no. 1, 15-53.

[4] R. de la Bretèche, Nombre de points de hauteur bornée sur les surfaces de del Pezzo de degré 5, Duke Math. J. 113 (2002), no. 3, 421-464.

[5] R. de la Bretèche and T. D. Browning, On Manin's conjecture for singular del Pezzo surfaces of degree four, I, Mich. Math. J., to appear (arXiv:math.NT/0412086, 2004)

[6] - On Manin's conjecture for singular del Pezzo surfaces of degree four, II, Math. Proc. Camb. Phil. Soc., to appear (arXiv:math.NT/0502510, 2005)

[7] R. de la Bretèche, T. D. Browning, and U. Derenthal, On Manin's conjecture for a certain singular cubic surface, Ann. Sci. École Norm. Sup. (4), to appear (arXiv:math.NT/0509370, 2005)

[8] R. de la Bretèche and E. Fouvry, L'éclaté du plan projectif en quatre points dont deux conjugués, J. Reine Angew. Math. 576 (2004) 63-122.

[9] T. D. Browning, An overview of Manin's conjecture for del Pezzo surfaces in proceedings of the Gauss-Dirichlet conference (Göttingen, 2005), Vol. 7 of Clay Math. Proc., 39-55, Amer. Math. Soc., Providence, RI, 2007.

[10] J. W. Bruce and C. T. C. Wall, On the classification of cubic surfaces, J. London Math. Soc. (2) 19 (1979), no. 2, 245-256.

[11] D. F. Coray and M. A. Tsfasman, Arithmetic on singular Del Pezzo surfaces, Proc. London Math. Soc. (3) 57 (1988), no. 1, 25-87.

[12] U. Derenthal, Singular Del Pezzo surfaces whose universal torsors are hypersurfaces (arXiv:math.AG/0604194, 2006).

[13] - Geometry of universal torsors (thesis, 2006). 


\begin{tabular}{|c||c|c|c|c|}
\hline type & singularities & lines & generators of $\Lambda_{\text {eff }}^{\vee}$ & $\alpha$ \\
\hline \hline 0 & - & 27 & 99 & $1 / 120$ \\
$i$ & $\mathbf{A}_{1}$ & 21 & 78 & $1 / 240$ \\
$i i$ & $2 \mathbf{A}_{1}$ & 16 & 62 & $1 / 480$ \\
iii & $\mathbf{A}_{2}$ & 15 & 52 & $1 / 720$ \\
$i v$ & $3 \mathbf{A}_{1}$ & 12 & 49 & $1 / 960$ \\
v & $\mathbf{A}_{2}+\mathbf{A}_{1}$ & 11 & 43 & $1 / 1440$ \\
vi & $\mathbf{A}_{3}$ & 10 & 32 & $1 / 2880$ \\
vii & $4 \mathbf{A}_{1}$ & 9 & 38 & $1 / 1920$ \\
viii & $\mathbf{A}_{2}+2 \mathbf{A}_{1}$ & 8 & 35 & $1 / 2880$ \\
ix & $\mathbf{A}_{3}+\mathbf{A}_{1}$ & 7 & 27 & $1 / 5760$ \\
$x$ & $2 \mathbf{A}_{2}$ & 7 & 31 & $1 / 4320$ \\
xi & $\mathbf{A}_{4}$ & 6 & 21 & $1 / 14400$ \\
xii & $\mathbf{D}_{4}$ & 6 & 16 & $1 / 23040$ \\
xiii & $\mathbf{A}_{3}+2 \mathbf{A}_{1}$ & 5 & 23 & $1 / 11520$ \\
xiv & $2 \mathbf{A}_{2}+\mathbf{A}_{1}$ & 5 & 18 & $1 / 28800$ \\
xv & $\mathbf{A}_{4}+\mathbf{A}_{1}$ & 4 & 18 & $1 / 28800$ \\
xvi & $\mathbf{A}_{5}$ & 3 & 13 & $1 / 86400$ \\
xvii & $\mathbf{D}_{5}$ & 3 & 11 & $1 / 230400$ \\
xviii & $3 \mathbf{A}_{2}$ & 3 & 21 & $1 / 25920$ \\
xix & $\mathbf{A}_{5}+\mathbf{A}_{1}$ & 2 & 13 & $1 / 172800$ \\
xx & $\mathbf{E}_{6}$ & 1 & 7 & $1 / 6220800$ \\
\hline
\end{tabular}

TABle 6. Del Pezzo surfaces of degree 3

[14] U. Derenthal and Y. Tschinkel, Universal torsors over Del Pezzo surfaces and rational points, in Equidistribution in Number Theory, An Introduction (Montréal, 2005), Vol. 237 of NATO Sci. Ser. II Math. Phys. Chem., 169-196, Springer, Dordrecht (2006).

[15] J. Franke, Y. I. Manin, and Y. Tschinkel, Rational points of bounded height on Fano varieties, Invent. Math. 95 (1989), no. 2, 421-435.

[16] R. Friedman and J. W. Morgan, Exceptional groups and del Pezzo surfaces, in Symposium in Honor of C. H. Clemens (Salt Lake City, UT, 2000), Vol. 312 of Contemp. Math., 101-116, Amer. Math. Soc., Providence, RI (2002).

[17] E. Gawrilow and M. Joswig, polymake: a framework for analyzing convex polytopes, in Polytopes - combinatorics and computation (Oberwolfach, 1997), Vol. 29 of DMV Sem., 4373, Birkhäuser, Basel (2000).

[18] E. Peyre, Hauteurs et mesures de Tamagawa sur les variétés de Fano, Duke Math. J. 79 (1995), no. $1,101-218$.

[19] E. Peyre and Y. Tschinkel, Tamagawa numbers of diagonal cubic surfaces of higher rank, in Rational points on algebraic varieties, Vol. 199 of Progr. Math., 275-305, Birkhäuser, Basel (2001).

Institut FÜr Mathematik, Universität ZürICH, Winterthurerstrasse 190, 8057 Zürich, SWITZERLAND

E-mail address: ulrich.derenthal@math.unizh.ch 